Supporting information for:

\title{
Asymmetric Synthesis of Four Isomers of 2-C-Trifluoro
}

\section{methylerythritol}

Hua Wang, Xiaoming Zhao, Youhua Li and Long Lu

1. General Experimental Method.

2. Chemical data of (12a-3-Mosher's ester) 12b, 12c, 12d, 12e, 12f, 12g, 12i, 13b, 13c, 13d. S3

3. ${ }^{13}$ C NMR spectra of $\mathbf{4 - 1 3}$

(Z)-2-Benzyloxymethyl-1,1,1-trifluoro-but-3-enoic acid ethyl ester (4) S8

(E)-2-Benzyloxymethyl-1,1,1-trifluoro-but-3-enoic acid ethyl ester (5).

(Z)-2-Benzyloxymethyl-1,1,1-trifluoro-but-3-en-4-ol (6)... $\mathrm{S} 10$

(Z)-Benzoic acid 2-benzyloxymethyl-1,1,1-trifluoro-but-3-enyl ester (7)....

(Z)-Benzoic acid 1,1,1-trifluoro-2-hydroxymethyl-but-3-enylester (8)...

(Z)-Benzoic acid 2-(tert-butyl-diphenyl-silanyloxymethyl)-1,1,1-trifluoro-but-3-enyl ester (9).....S13

(Z)-Benzoic acid 1,1,1-trifluoro-2-trityloxymethyl-but-3-enyl ester (10) S14

$(2 R, 3 S)$-2-Benzyloxymethyl-1,1,1-trifluoro-2,3-dihydroxy-butyric acid ethyl ester (12a)..... S15

(2S, 3S)-2-Benzyloxymethyl-1,1,1-trifluoro-2,3-dihydroxy-butyric acid ethyl ester (12b) S16 
1-(Benzyloxy)-2-(trifluoromethyl)butane-2,3,4-triol (12c).

Benzoic acid 2-(tert-butyl-diphenyl-silanyloxymethyl)-1,1,1-trifluoro-2,3-dihydroxy-butyl ester (12f). $\mathrm{S} 18$

Benzoic acid -1,1,1-trifluoro-2,3-dihydroxy-2-trityloxymethyl butyl ester (12g) S19

(2S, 3R)-2-Benzyloxymethyl-1,1,1-trifluoro-2,3-dihydroxy-butyric acid ethyl ester (12h) S20

$(2 R, 3 R)$-2-Benzyloxymethyl-1,1,1-trifluoro-2,3-dihydroxy-butyric acid ethyl ester (12i) $\mathrm{S} 21$

(2R, 3R)-2-C-Trifluoromethylerythritol (13a) $\mathrm{S} 22$

(2S, 3R)-2-C-Trifluoromethylerythritol (13b) S23

(2S, 3S)-2-C-Trifluoromethylerythritol (13c) S24

(2R, 3S)-2-C-Trifluoromethylerythritol (13d) . $\mathrm{S} 25$

4. ${ }^{1} H$ NMR spectra of 12a-3-Mosher's ester. S26

(R)-12a-3-Mosher's ester. S26

(S)-12a-3-Mosher's ester. S27

5. An ORTEP view of $\mathbf{1 2 a}$ S28 


\section{General Experimental Methods}

${ }^{1} \mathrm{H}$ NMR, ${ }^{19} \mathrm{~F}$ NMR and ${ }^{13} \mathrm{C}$ NMR spectra were recorded at $25{ }^{\circ} \mathrm{C}$. Chemical shifts $(\delta)$ are quoted in ppm and referenced to internal TMS $(\delta 0)$ for ${ }^{1} \mathrm{H}$ NMR, and $\mathrm{CDCl}_{3}(\delta 77.0)$ or $\mathrm{MeOH}-\mathrm{d}_{4}(\delta 49.0)$ for ${ }^{13} \mathrm{C}$ NMR; coupling constants $(J)$ are quoted in Hz; data are reported as follows: s, singlet; d, doublet; t, triplet; q, quartet; m, multiplet; br, broad; Flash chromatography was performed on silica gel (300-400 mesh), All reactions were conducted in oven-dried glassware under inert atmosphere of nitrogen with anhydrous solvents. The solvents and reagents were purified and dried according to standard procedures.

2. Chemical data of (12a Mosher's ester), 12b, 12c, 12d, 12e, 12f, 12g, 12i, 13b, 13c, $13 d$.

(R)-12a-3-Mosher's ester. Oil, in 67\% yield. ${ }^{1} \mathrm{H}$ NMR $\left(\mathrm{CDCl}_{3}\right) \delta 1.16(\mathrm{t}, 3 \mathrm{H}, J=7.2 \mathrm{~Hz}), 3.51$ (d, $3 \mathrm{H}, J=1.2 \mathrm{~Hz}), 3.71(\mathrm{~d}, 1 \mathrm{H}, J=9.9 \mathrm{~Hz}), 3.83(\mathrm{~d}, 1 \mathrm{H}, J=9.9 \mathrm{~Hz}), 4.01 \sim 4.10(\mathrm{~m}, 2 \mathrm{H}), 4.44 \sim 4.53(\mathrm{~m}$, 3H), $5.47(\mathrm{~s}, 1 \mathrm{H}) 7.25 \sim 7.56(\mathrm{~m}, 10 \mathrm{H}) ;{ }^{13} \mathrm{C} \mathrm{NMR}\left(\mathrm{CDCl}_{3}\right) \delta 13.7(\mathrm{~s}), 29.7(\mathrm{~s}), 55.4(\mathrm{~s}), 62.8(\mathrm{~s}), 67.2(\mathrm{~s})$, 70.9 (s), 74.2 (s), 75.4 (q, $J=27.5 \mathrm{~Hz}), 84.8(\mathrm{q}, J=29.4 \mathrm{~Hz}), 123.1(\mathrm{q}, J=288.7 \mathrm{~Hz}), 123.7(\mathrm{q}, J=$ $286.2 \mathrm{~Hz}), 127.4$ (s), 127.9 (s), 128.2 (s), 128.4 (s), 128.5 (s), 129.4 (s), 131.4 (s), 136.7 (s), 165.4 (s), $166.5(\mathrm{~s}) ;{ }^{19} \mathrm{~F} \mathrm{NMR}\left(\mathrm{CDCl}_{3}\right) \delta-71.9(\mathrm{~s}),-78.0(\mathrm{~s})$; IR (thin film) $v_{\max } 3512,3069,1760,1454,1187$, $1108 \mathrm{~cm}^{-1}$; MS (ESI): $\mathrm{m} / z$ $539.2\left(\mathrm{M}+\mathrm{H}^{+}\right)$. HRMS $\left(\mathrm{M}+\mathrm{Na}^{+}\right)$Anals. Calc'd for $\mathrm{C}_{24} \mathrm{H}_{24} \mathrm{O}_{7} \mathrm{~F}_{6} \mathrm{Na} 561.1319$; Found: 561.1318. $[\alpha]^{20}{ }_{\mathrm{D}}+9.1\left(c 1.5, \mathrm{CDCl}_{3}\right)$.

(S)-12a-3-Mosher's ester. Oil, in 39\% yield. ${ }^{1} \mathrm{H}$ NMR $\left(\mathrm{CDCl}_{3}\right) \delta 1.17(\mathrm{t}, 3 \mathrm{H}, J=7.2 \mathrm{~Hz}), 3.61$ (d, $3 \mathrm{H}, J=1.2 \mathrm{~Hz}), 3.67(\mathrm{~d}, 1 \mathrm{H}, J=9.9 \mathrm{~Hz}), 3.85(\mathrm{~d}, 1 \mathrm{H}, J=9.9 \mathrm{~Hz}), 4.00 \sim 4.20(\mathrm{~m}, 2 \mathrm{H}), 4.38(\mathrm{~s}, 1 \mathrm{H}), 4.46$ $(\mathrm{d}, 1 \mathrm{H}, J=11.7 \mathrm{~Hz}), 4.52(\mathrm{~d}, 1 \mathrm{H}, J=10.8 \mathrm{~Hz}), 5.47(\mathrm{~s}, 1 \mathrm{H}), 7.25 \sim 7.44(\mathrm{~m}, 8 \mathrm{H}), 7.58 \sim 7.60(\mathrm{~m}, 2 \mathrm{H}) ;{ }^{13} \mathrm{C}$ $\operatorname{NMR}\left(\mathrm{CDCl}_{3}\right) \delta 13.7(\mathrm{~s}), 29.7(\mathrm{~s}), 55.6(\mathrm{~s}), 62.8(\mathrm{~s}), 67.2(\mathrm{~s}), 70.8(\mathrm{~s}), 74.2(\mathrm{~s}), 75.5$ (q, $\left.J=26.7 \mathrm{~Hz}\right)$, $84.6(\mathrm{q}, J=27.7 \mathrm{~Hz}), 123.1(\mathrm{q}, J=287.2 \mathrm{~Hz}), 123.6(\mathrm{q}, J=284.8 \mathrm{~Hz}), 127.4(\mathrm{~s}), 127.9$ (s), $128.2(\mathrm{~s})$, $128.4(\mathrm{~s}), 128.5$ (s), 128.8 (s), 131.7 (s), 136.7 (s), 165.3 (s), 167.2 (s); ${ }^{19} \mathrm{~F}$ NMR $\left(\mathrm{CDCl}_{3}\right) \delta-72.0(\mathrm{~s})$, -78.3 (s); IR (thin film) $v_{\max } 3505,3068,2987,1759,1454,1187,1108 \mathrm{~cm}^{-1}$; MS (ESI): $\mathrm{m} / z 539.2$ 
$\left(\mathrm{M}+\mathrm{H}^{+}\right)$. HRMS $\left(\mathrm{M}+\mathrm{Na}^{+}\right)$Anals. Calc'd for $\mathrm{C}_{24} \mathrm{H}_{24} \mathrm{O}_{7} \mathrm{~F}_{6} \mathrm{Na} 561.1319$; Found: 561.1318. $[\alpha]^{20}{ }_{\mathrm{D}}-17.9$ $\left(c 1.2, \mathrm{CDCl}_{3}\right)$.

(2S, 3S)-2-Benzyloxymethyl-1,1,1-trifluoro-2,3-dihydroxy-butyric acid ethyl ester (12b). Compound 12b was purified on silica gel (PE: EtOAc $\left.=5: 1, R_{f}=0.4\right)$ in $77 \%$ yield as solid. mp: 35.5 $36{ }^{\circ} \mathrm{C} .{ }^{1} \mathrm{H}$ NMR $\left(\mathrm{CDCl}_{3}\right) \delta 1.30(\mathrm{t}, 3 \mathrm{H}, J=7.2 \mathrm{~Hz}), 3.29(\mathrm{br}, 1 \mathrm{H}), 3.80(\mathrm{~d}, 1 \mathrm{H}, J=10.2 \mathrm{~Hz}), 3.84(\mathrm{~d}, 1 \mathrm{H}$, $J=10.2 \mathrm{~Hz}), 4.00(\mathrm{br}, 1 \mathrm{H}), 4.20 \sim 4.33(\mathrm{~m}, 2 \mathrm{H}), 4.51(\mathrm{~s}, 1 \mathrm{H}), 4.62(\mathrm{~s}, 1 \mathrm{H}), 7.30 \sim 7.40(\mathrm{~m}, 5 \mathrm{H}) .{ }^{13} \mathrm{C} \mathrm{NMR}$ $\left(\mathrm{CDCl}_{3}\right) \delta 13.9(\mathrm{~s}), 62.8(\mathrm{~s}), 68.2(\mathrm{q}, J=2.3 \mathrm{~Hz}), 70.4(\mathrm{~s}), 74.2(\mathrm{~s}) 76.4(\mathrm{q}, J=26.2 \mathrm{~Hz}), 124.5(\mathrm{q}, J=$ $285.2 \mathrm{~Hz}), 127.8$ (s), 128.1 (s), 128.5 (s), 137.1 (s), 171.4 (s). ${ }^{19} \mathrm{~F} \mathrm{NMR}\left(\mathrm{CDCl}_{3}\right) \delta-76.2$ (t, $\left.J=7.1 \mathrm{~Hz}\right)$; IR (thin film) $v_{\max } 3476,2897,1738,1277,1188,1110 \mathrm{~cm}^{-1}$; MS (EI): $\mathrm{m} / 2322\left(\mathrm{M}^{+}, 0.8\right)$. Anals. Calc'd for $\mathrm{C}_{14} \mathrm{H}_{17} \mathrm{O}_{5} \mathrm{~F}_{3}$ : C, 52.17\%; H, 5.28\%. Found: C, 52.14\%; H, 5.31\%. $[\alpha]^{20}{ }_{\mathrm{D}}+4.6\left(c 0.84, \mathrm{CHCl}_{3}\right) \cdot \mathrm{t}_{\mathrm{r}}$ $(2 \mathrm{~S}, 3 \mathrm{~S})=17.14 \mathrm{~min}_{\mathrm{r}}(2 \mathrm{R}, 3 \mathrm{R})=20.10 \mathrm{~min},($ Chiralpak OJ, column No. OJ00CE-HI043, $\lambda=254 \mathrm{~nm}$, Hex: $i-\mathrm{PrOH}=90: 10,0.7 \mathrm{~mL} / \mathrm{min})$.

1-(Benzyloxy)-2-(trifluoromethyl)butane-2,3,4-triol (12c). Oil, in 81\% yield. ${ }^{1} \mathrm{H}$ NMR $\left(\mathrm{CDCl}_{3}\right) \delta$ 2.29 (s), 3.07 (s), $3.61 \sim 3.79(\mathrm{~m}, 5 \mathrm{H}), 4.01(\mathrm{~s}), 4.19(\mathrm{~s}), 4.55(\mathrm{~s}, 2 \mathrm{H}), 7.28 \sim 7.38(\mathrm{~m}, 5 \mathrm{H}) ;{ }^{13} \mathrm{C}$ NMR $\left(\mathrm{CDCl}_{3}\right) \delta 61.9(\mathrm{~s}), 68.1$ (s), $71.4(\mathrm{~s}), 74.1(\mathrm{~s}) 76.5(\mathrm{q}, J=26.3 \mathrm{~Hz}), 125.0(\mathrm{q}, J=285.1 \mathrm{~Hz}), 127.8(\mathrm{~s})$, $128.2(\mathrm{~s}), 128.6(\mathrm{~s}), 136.7(\mathrm{~s}) ;{ }^{19} \mathrm{~F}$ NMR $\left(\mathrm{CDCl}_{3}\right) \delta-76.5(\mathrm{~s}) ; \mathrm{IR}$ (thin film) $v_{\max } 3420,2936,1456,1182$, 1151, $1095 \mathrm{~cm}^{-1}$; MS (ESI): $m / z 298.1\left(\mathrm{M}+\mathrm{NH}_{4}{ }^{+}\right)$. HRMS $\left(\mathrm{M}+\mathrm{Na}^{+}\right)$Anals. Calc'd for $\mathrm{C}_{12} \mathrm{H}_{15} \mathrm{O}_{4} \mathrm{~F}_{3} \mathrm{Na}$ 303.0820 Found: $303.0822 .[\alpha]^{20}{ }_{\mathrm{D}}+4.3\left(c 0.55, \mathrm{CHCl}_{3}\right)$

2-((Benzyloxy)methyl)-1,1,1-trifluoro-2,3-dihydroxybutyl benzoate (12d). Oil, in $81 \%$ yield. ${ }^{1} \mathrm{H}$ $\operatorname{NMR}\left(\mathrm{CDCl}_{3}\right) \delta 3.14(\mathrm{~d}, 1 \mathrm{H}, J=5.4 \mathrm{~Hz}), 3.76(\mathrm{~d}, 1 \mathrm{H}, J=10.2 \mathrm{~Hz}), 3.87(\mathrm{~d}, 1 \mathrm{H}, J=10.2 \mathrm{~Hz}), 3.92(\mathrm{~s}$, 1H), 4.27 4.32 (br, 1H), 4.42 4.49 (m, 1H), 4.58 4.63 (m, 3H), 7.29 7.38 (m, 5H), $7.42(\mathrm{t}, 2 \mathrm{H}, J=7.5$ $\mathrm{Hz}), 7.56(\mathrm{t}, 1 \mathrm{H}, J=7.5 \mathrm{~Hz}), 8.01(\mathrm{~d} 2 \mathrm{H}, J=7.5 \mathrm{~Hz}) ;{ }^{13} \mathrm{C} \mathrm{NMR}\left(\mathrm{CDCl}_{3}\right) \delta 64.8(\mathrm{~s}), 68.2(\mathrm{~s}), 70.8(\mathrm{~s})$, $74.2(\mathrm{~s}), 75.3(\mathrm{q}, J=26.5 \mathrm{~Hz}), 124.7(\mathrm{q}, J=285.0 \mathrm{~Hz}), 127.8(\mathrm{~s}), 128.2(\mathrm{~s}), 128.5(\mathrm{~s}), 128.6(\mathrm{~s}), 129.6$ (s), $129.7(\mathrm{~s}), 133.3(\mathrm{~s}), 136.7(\mathrm{~s}), 166.9(\mathrm{~s}) ;{ }^{19} \mathrm{~F} \mathrm{NMR}\left(\mathrm{CDCl}_{3}\right) \delta-76.2$ (s); IR (thin film) $v_{\max }$ 3456, 
3399, 2905, 1704, 1291, $1191 \mathrm{~cm}^{-1}$; MS (EI): $\mathrm{m} / z 384\left(\mathrm{M}^{+}, 0.2\right)$. Anals. Calc'd for $\mathrm{C}_{19} \mathrm{H}_{19} \mathrm{O}_{5} \mathrm{~F}_{3}$ : C, 59.38\%; H, 4.98\%. Found: C, 59.42\%; H, 5.08\%.

2-(Trifluoromethyl)-1,2,3-trihydroxybutyl benzoate (12e). Solid, in 76\% yield. mp: $139-140{ }^{\circ} \mathrm{C}$. ${ }^{1} \mathrm{H}$ NMR $\left(\mathrm{CDCl}_{3}\right) \delta 3.84(\mathrm{~d}, 1 \mathrm{H}, J=11.7 \mathrm{~Hz}), 4.00(\mathrm{~d}, 2 \mathrm{H}, J=11.7 \mathrm{~Hz}), 4.38 \sim 4.50(\mathrm{~m}, 2 \mathrm{H}), 4.69 \sim 4.73$ $(\mathrm{m}, 1 \mathrm{H}), 7.51(\mathrm{t}, 2 \mathrm{H}, J=7.5 \mathrm{~Hz}), 7.64(\mathrm{t}, 1 \mathrm{H}, J=7.5 \mathrm{~Hz}), 8.08(\mathrm{~d}, 2 \mathrm{H}, J=7.5 \mathrm{~Hz}) ;{ }^{13} \mathrm{C} \mathrm{NMR}\left(\mathrm{CDCl}_{3}\right) \delta$ $61.1(\mathrm{~s}), 65.7$ (s), $70.0(\mathrm{~s}), 75.7(\mathrm{q}, J=24.37 \mathrm{~Hz}), 126.1$ (q, $J=284.9 \mathrm{~Hz}), 128.4(\mathrm{~s}), 129.5(\mathrm{~s}), 130.4(\mathrm{~s})$, $133.0(\mathrm{~s}) ;{ }^{19} \mathrm{~F}$ NMR $\left(\mathrm{CDCl}_{3}\right) \delta-75.2$ (s); IR (thin film) v $v_{\max } 3376,2966,1703,1293,1176 \mathrm{~cm}^{-1}$; MS (EI): $m / z 165\left(\mathrm{M}_{-} \mathrm{C}_{3} \mathrm{H}_{4} \mathrm{~F}_{3} \mathrm{O}_{2}{ }^{+}, 72.2\right)$. Anals. Calc'd for $\mathrm{C}_{12} \mathrm{H}_{13} \mathrm{~F}_{3} \mathrm{O}_{5}$ : C, 48.99\%; H, 4.45\%. Found: C, 48.63\%; H, $4.56 \%$.

\section{Benzoic acid 2-(tert-butyl-diphenyl-silanyloxymethyl)-1,1,1-trifluoro-2,3-dihydroxy-butyl ester} (12f). Oil, in 81\% yield. ${ }^{1} \mathrm{H}$ NMR $\left(\mathrm{CDCl}_{3}\right) \delta 1.10$ (s, 9H), $2.94(\mathrm{br}, 1 \mathrm{H}), 3.76(\mathrm{~s}, 1 \mathrm{H}), 3.97$ (q, 2H, $J=$ $11.4 \mathrm{~Hz}), 4.31 \sim 4.33$ (br, 1H), 4.47 4.67 (m, 2H), 7.40 7.49 (m, 8H), 7.54 7.60 (m, 1H), 7.66 7.70 (m, 4H), 8.00 8.03 (m, 2H); ${ }^{13} \mathrm{C} \mathrm{NMR}\left(\mathrm{CDCl}_{3}\right) \delta 19.3(\mathrm{~s}), 26.8$ (s), 62.3 (s), 65.4 (s), 70.0 (s), 75.5 (q, $J=$ $26.1 \mathrm{~Hz}), 125.3(\mathrm{q}, J=259.4 \mathrm{~Hz}), 128.1(\mathrm{~s}), 128.5(\mathrm{~s}), 129.6(\mathrm{~s}), 130.2(\mathrm{~s}), 131.9(\mathrm{~s}), 132.0$ (s), 133.3 (s), $135.6(\mathrm{~s}) ;{ }^{19} \mathrm{~F}$ NMR $\left(\mathrm{CDCl}_{3}\right) \delta-76.3$ (s); IR (thin film) $v_{\max } 3482,2869,1724,1707,1278,1180,1114$ $\mathrm{cm}^{-1}$; MS (ESI): $\mathrm{m} / z 532.4\left(\mathrm{M}+\mathrm{H}^{+}\right)$. HRMS. $\left(\mathrm{M}+\mathrm{Na}^{+}\right)$Anals. Calc'd for $\mathrm{C}_{28} \mathrm{H}_{31} \mathrm{~F}_{3} \mathrm{O}_{5} \mathrm{SiNa}$ : 555.1780 . Found: 555.1785 .

Benzoic acid -1,1,1-trifluoro-2,3-dihydroxy-2-trityloxymethyl butyl ester (12g). White solid, in 83\% yield. mp: $108.5-110{ }^{\circ} \mathrm{C} .{ }^{1} \mathrm{H}$ NMR $\left(\mathrm{CDCl}_{3}\right) \delta 2.72(\mathrm{~d}, 1 \mathrm{H}, J=4.8 \mathrm{~Hz}), 3.46 \sim 3.67(\mathrm{~m}, 2 \mathrm{H})$, 4.26 4.48 (m, 2H), 7.19 7.57 (m, 13H), 7.95 7.98 (m, 2H). ${ }^{13} \mathrm{C} \mathrm{NMR}\left(\mathrm{CDCl}_{3}\right) \delta 61.6(\mathrm{~s}), 65.3(\mathrm{~s}), 70.3$ (s), $75.6(\mathrm{q}, J=28.1 \mathrm{~Hz}), 88.1(\mathrm{~s}), 125.2$ (q, $J=286.3 \mathrm{~Hz}), 127.6(\mathrm{~s}), 128.0(\mathrm{~s}), 128.2$ (s), 128.5 (s), $128.6(\mathrm{~s}), 129.7$ (s), 130.9 (s), 142.9 (s), 166.9 (s); ${ }^{19} \mathrm{~F}$ NMR $\left(\mathrm{CDCl}_{3}\right) \delta-75.5$ (s); IR (thin film) $v_{\max }$ 3349, 3061, 2846, 1701, 1601, 1450, 1179, $1063 \mathrm{~cm}^{-1}$; MS (ESI): $m / z 559.2\left(\mathrm{M}+\mathrm{Na}^{+}\right)$. HRMS.(M+Na $\left.{ }^{+}\right)$ Anals. Calc'd for $\mathrm{C}_{31} \mathrm{H}_{27} \mathrm{O}_{5} \mathrm{~F}_{3} \mathrm{Na}$ : 559.1706. Found: 559.1702. 
(2S, 3R)-2-Benzyloxymethyl-1,1,1-trifluoro-2,3-dihydroxy-butyric acid ethyl ester (12h). Oil, in $81 \%$ yield. ${ }^{1} \mathrm{H}$ NMR $\left(\mathrm{CDCl}_{3}\right) \delta 1.31(\mathrm{t}, 3 \mathrm{H}, J=7.2 \mathrm{~Hz}), 3.26(\mathrm{~d}, 1 \mathrm{H}, J=7.2 \mathrm{~Hz}), 3.75(\mathrm{~d}, 1 \mathrm{H}, J=10.2$ $\mathrm{Hz}), 3.92(\mathrm{~d}, 1 \mathrm{H}, J=10.2 \mathrm{~Hz}), 3.94(\mathrm{~s}, 1 \mathrm{H}), 4.25 \sim 4.34(\mathrm{~m}, 2 \mathrm{H}), 4.51(\mathrm{~d}, 1 \mathrm{H} J=6.9 \mathrm{~Hz}), 4.64(\mathrm{~s}, 2 \mathrm{H})$ 7.31 7.40 (m, 5H); ${ }^{13} \mathrm{C} \mathrm{NMR}\left(\mathrm{CDCl}_{3}\right) \delta 13.9(\mathrm{~s}), 62.9$ (s), $68.1(\mathrm{~s}), 70.4(\mathrm{~s}), 74.2(\mathrm{~s}), 76.4(\mathrm{q}, J=31.8$ $\mathrm{Hz}), 126.5(\mathrm{q}, J=283.6 \mathrm{~Hz}), 127.8(\mathrm{~s}), 128.1(\mathrm{~s}), 128.5(\mathrm{~s}), 137.0(\mathrm{~s}), 171.5(\mathrm{~s}) ;{ }^{19} \mathrm{~F} \mathrm{NMR}\left(\mathrm{CDCl}_{3}\right) \delta-$ 76.5 (s); IR (thin film) $v_{\max } 3489,3428,2901,1740,1251,1098 \mathrm{~cm}^{-1}$; MS (ESI): $\mathrm{m} / z 323.0\left(\mathrm{M}+\mathrm{H}^{+}\right)$. HRMS. $\left(\mathrm{M}+\mathrm{Na}^{+}\right)$Anals. Calc'd for $\mathrm{C}_{14} \mathrm{H}_{17} \mathrm{O}_{5} \mathrm{~F}_{3} \mathrm{Na}$ : 345.0925, Found: 345.0920. $[\alpha]^{20}{ }_{\mathrm{D}}-21.3(c 0.75$, $\left.\mathrm{CHCl}_{3}\right) .(82 \%$ ee $) . \mathrm{t}_{\mathrm{r}}(2 \mathrm{R}, 3 \mathrm{~S})=20.50 \mathrm{~min}_{\mathrm{r}}(2 \mathrm{~S}, 3 \mathrm{R})=21.44 \mathrm{~min},($ Chiralpak AS, column No. AS00CEJG019, $\lambda=214 \mathrm{~nm}$, Hex: $i-\mathrm{PrOH}=90: 10,0.7 \mathrm{~mL} / \mathrm{min})$.

$(2 R, 3 R)$-2-Benzyloxymethyl-1,1,1-trifluoro-2,3-dihydroxy-butyric acid ethyl ester (12i). Oil, in $79 \%$ yield. ${ }^{1} \mathrm{H}$ NMR $\left(\mathrm{CDCl}_{3}\right) \delta 1.21(\mathrm{t}, 3 \mathrm{H}, J=7.5 \mathrm{~Hz}), 3.35(\mathrm{~d}, 1 \mathrm{H}, J=6.6 \mathrm{~Hz}), 3.72(\mathrm{~d}, 1 \mathrm{H}, J=9.9$ $\mathrm{Hz}), 3.89(\mathrm{~d}, 1 \mathrm{H}, J=10.2 \mathrm{~Hz}), 4.08(\mathrm{~s}, 1 \mathrm{H}), 4.07 \sim 4.20(\mathrm{~m}, 2 \mathrm{H}), 4.48(\mathrm{~d}, 1 \mathrm{H}, J=6.6 \mathrm{~Hz}), 4.53(\mathrm{~d}, 1 \mathrm{H}, J$ $=11.7 \mathrm{~Hz}), 4.60(\mathrm{~d}, 1 \mathrm{H}, J=11.1 \mathrm{~Hz}), 7.31 \sim 7.39(\mathrm{~m}, 5 \mathrm{H}) ;{ }^{13} \mathrm{C} \mathrm{NMR}\left(\mathrm{CDCl}_{3}\right) \delta 13.8(\mathrm{~s}), 62.9(\mathrm{~s}), 67.2$ (s), $69.2(\mathrm{~s}), 74.2$ (s), 76.3 (q, $J=26.4 \mathrm{~Hz}), 124.3$ (q, $J=284.8 \mathrm{~Hz}), 128.1$ (s), $128.2(\mathrm{~s}), 128.5$ (s), 136.9 (s), $171.6(\mathrm{~s}) ;{ }^{19} \mathrm{~F}$ NMR $\left(\mathrm{CDCl}_{3}\right) \delta-77.4(\mathrm{~s})$; IR (thin film) $v_{\max } 3474,2941,1737,1278,1188,1154 \mathrm{~cm}^{-1}$ ; MS (ESI): $m / z$ 323.2. $\left(\mathrm{M}+\mathrm{H}^{+}\right)$. HRMS. $\left(\mathrm{M}+\mathrm{Na}^{+}\right)$Anals. Calc'd for $\mathrm{C}_{14} \mathrm{H}_{17} \mathrm{O}_{5} \mathrm{~F}_{3} \mathrm{Na}$ : 345.0925 . Found: 345.0920. $[\alpha]^{20}{ }_{\mathrm{D}}-3.5\left(c 0.90, \mathrm{CHCl}_{3}\right)(50 \%$ ee $) . \mathrm{t}_{\mathrm{r}}(2 \mathrm{~S}, 3 \mathrm{~S})=31.14 \min \mathrm{t}_{\mathrm{r}}(2 \mathrm{R}, 3 \mathrm{R})=36.64 \mathrm{~min}$, (Chiralpak AS, column No. AS00CE-JG019, $\lambda=220$ nm, Hex: $i$-PrOH = 95:5, $0.7 \mathrm{~mL} / \mathrm{min}$ ).

(2S, 3R)-2-C-Trifluoromethylerythritol (13b). Oil, in 92\% yield. ${ }^{1} \mathrm{H}$ NMR $\left(\mathrm{MeOH}-\mathrm{d}_{4}\right) \delta$ 3.60 3.73 (m, 2H), 3.82 3.89 (m, 2H), 3.95 3.98 (m, 1H); ${ }^{13} \mathrm{C} \mathrm{NMR} \mathrm{(MeOH-d_{4 } ) \delta 6 1 . 0 ( s ) , ~} 61.9(\mathrm{~s})$, 71.9 (s), 75.9 (q, $J=24.8 \mathrm{~Hz}), 125.9(\mathrm{q}, J=286.9 \mathrm{~Hz}) ;{ }^{19} \mathrm{~F}$ NMR $\left(\mathrm{MeOH}_{4}\right) \delta-76.5$ (s); IR (thin film) $v_{\max } 3401,2960,1639,1181,1099,1054 \mathrm{~cm}^{-1}$; MS (ESI): $m / z 208.1\left(\mathrm{M}+\mathrm{NH}_{4}^{+}\right) . \mathrm{HRMS}\left(\mathrm{M}+\mathrm{Na}^{+}\right)$Anals. Calc'd for $\mathrm{C}_{5} \mathrm{H}_{9} \mathrm{O}_{4} \mathrm{~F}_{3} \mathrm{Na}$ : 213.0352. Found: 213.0345. $[\alpha]^{20}{ }_{\mathrm{D}}+6.3(c$ 1.2, $\mathrm{MeOH})$.

(2S, 3S)-2-C-Trifluoromethylerythritol (13c). Oil, in 94\% yield. ${ }^{1} \mathrm{H}$ NMR $\left(\mathrm{MeOH}-\mathrm{d}_{4}\right) \delta$ 3.74 3.84 (m, 4H), 3.92 3.94 (m, 1H); ${ }^{13} \mathrm{C} \mathrm{NMR}\left(\mathrm{MeOH}-\mathrm{d}_{4}\right) \delta 60.3(\mathrm{~s}), 62.1(\mathrm{~s}), 70.9(\mathrm{~s}), 76.1(\mathrm{q}, J=$ 
$24.3 \mathrm{~Hz}), 126.2(\mathrm{q}, J=285.8 \mathrm{~Hz}) ;{ }^{19} \mathrm{~F}$ NMR $\left(\mathrm{MeOH}_{-} \mathrm{d}_{4}\right) \delta-77.6(\mathrm{~s})$; IR (thin film) $v_{\max } 3450,2956$, 1419, 1238, 1185, $1043 \mathrm{~cm}^{-1}$; MS (ESI): $m / z 191.1\left(\mathrm{M}+\mathrm{H}^{+}\right)$. HRMS $\left(\mathrm{M}+\mathrm{Na}^{+}\right)$Anals. Calc'd for $\mathrm{C}_{5} \mathrm{H}_{9} \mathrm{O}_{4} \mathrm{~F}_{3} \mathrm{Na}: 213.0352$. Found: 213.0345. $[\alpha]^{20}{ }_{\mathrm{D}}-10.2(c \mathrm{c} 0.55, \mathrm{MeOH})$.

(2R, 3S)-2-C-Trifluoromethylerythritol (13d). Oil, in $89 \%$ yield. ${ }^{1} \mathrm{H}$ NMR $\left(\mathrm{MeOH}-\mathrm{d}_{4}\right) \delta$ 3.61 3.73 (m, 2H), 3.83 3.88 (m, 2H), 3.95 3.98 (m, 1H); $\left.{ }^{13} \mathrm{C} \mathrm{NMR} \mathrm{(MeOH-d} 4\right) \delta 60.9(\mathrm{~s}), 61.8(\mathrm{~s})$, $71.8(\mathrm{~s}), 75.9(\mathrm{q}, J=24.8 \mathrm{~Hz}), 125.9(\mathrm{q}, J=285.4 \mathrm{~Hz}) ;{ }^{19} \mathrm{~F} \mathrm{NMR}\left(\mathrm{MeOH}-\mathrm{d}_{4}\right) \delta-76.5(\mathrm{~s})$; IR (thin film) $v_{\max } 3379,2959,1418,1179,1137,1110,1028 \mathrm{~cm}^{-1}$; MS (ESI): $\mathrm{m} / z 208.1\left(\mathrm{M}+\mathrm{NH}_{4}^{+}\right) . \mathrm{HRMS}\left(\mathrm{M}+\mathrm{Na}^{+}\right)$ Anals. Calc'd for $\mathrm{C}_{5} \mathrm{H}_{9} \mathrm{O}_{4} \mathrm{~F}_{3} \mathrm{Na}$ : 213.0352. Found: 213.0345. $[\alpha]^{20}{ }_{\mathrm{D}}-1.3(c 1.0, \mathrm{MeOH})$. 
$\vec{\circ}$

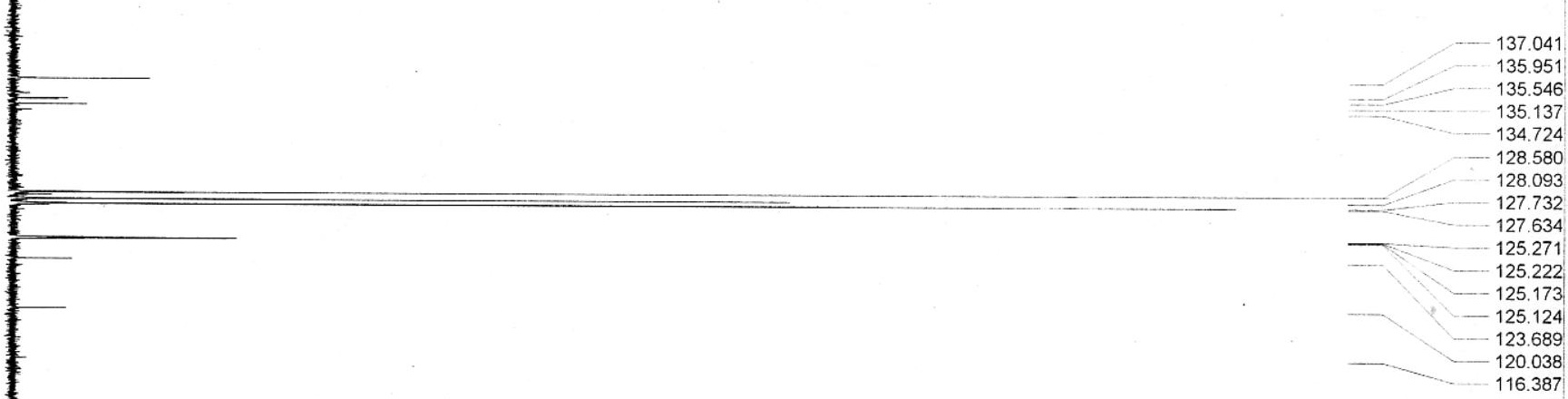

8.

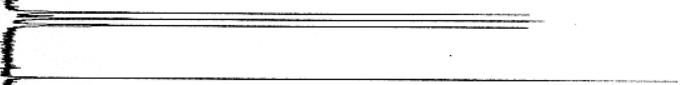<smiles>[O]C/C([O])=C/O[Hg]</smiles> 

128.905

128.876

128.590

128.505

128.459

128.293

128.170

128.091

28.07

127.921

127.808

127.744

127.345

126.952

126.928

126.560

126.532

126.160

125.226

125.226

124.773

21.578

117.923

107.265

79.877

77.674

77.645

77.595

77.460

$\begin{array}{r}77.366 \\ -77.305 \\ -77.239 \\ \hline\end{array}$

$\begin{array}{r}77.366 \\ -77.305 \\ \hline \\ \hline\end{array} \quad 77.239$

$\begin{array}{r}77.037 \\ -\quad 76.239 \\ \hline\end{array}$

76.940

$\begin{array}{r}76.879 \\ -\quad 76.860 \\ \hline-76.772\end{array}$

76.860
-76.772

76.610

72.551

68.324

68.285

68.28

68.243

68.207

58.914

58.883

58.847

58.811

41.646

37.787

29.853

29.730

ळึ

29.706

$-0.007$ 
咅

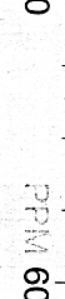

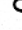

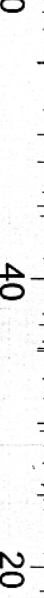




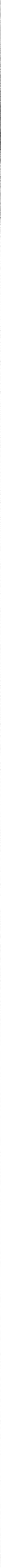




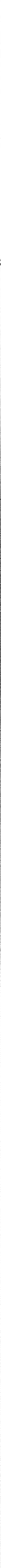




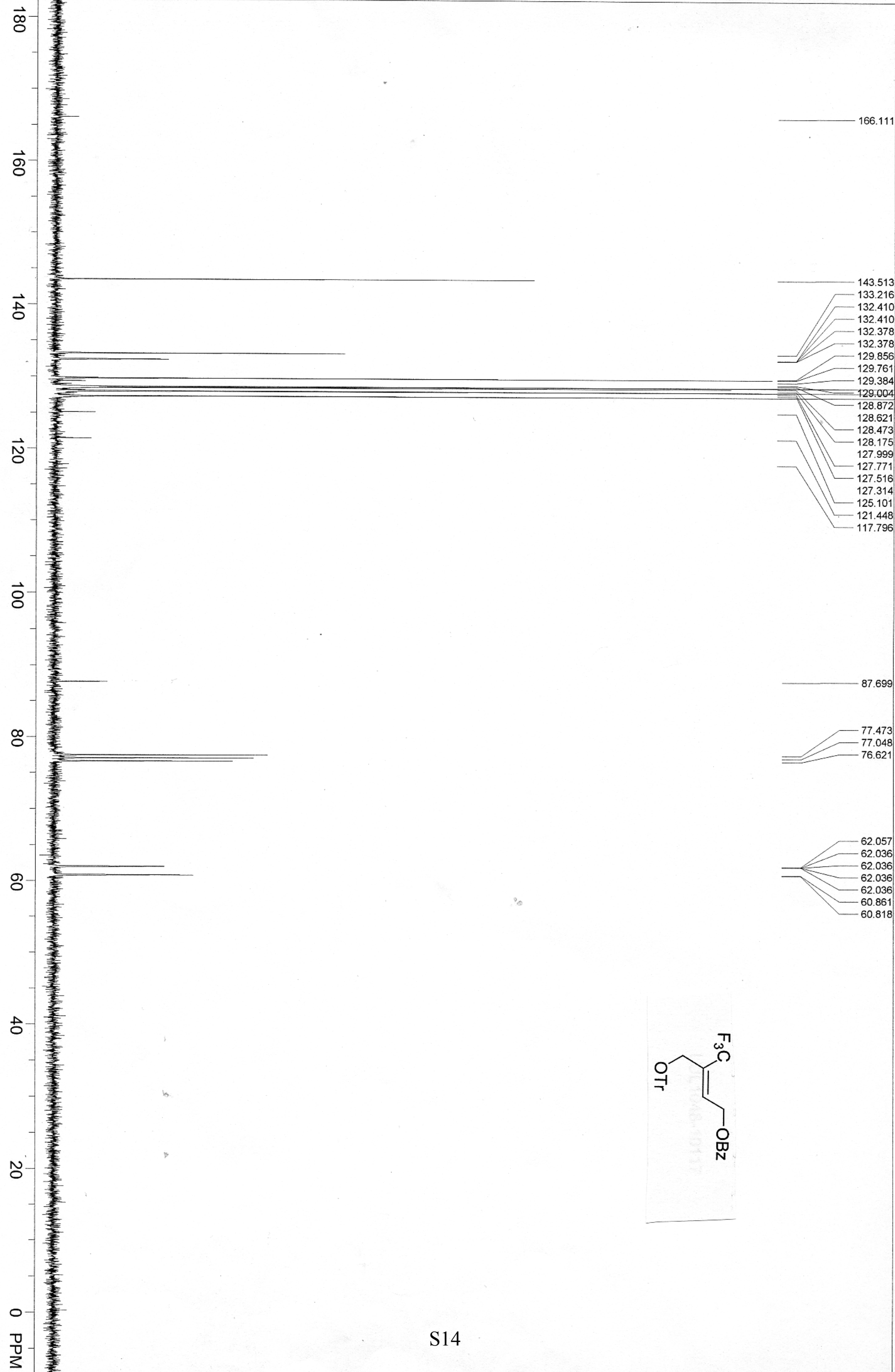




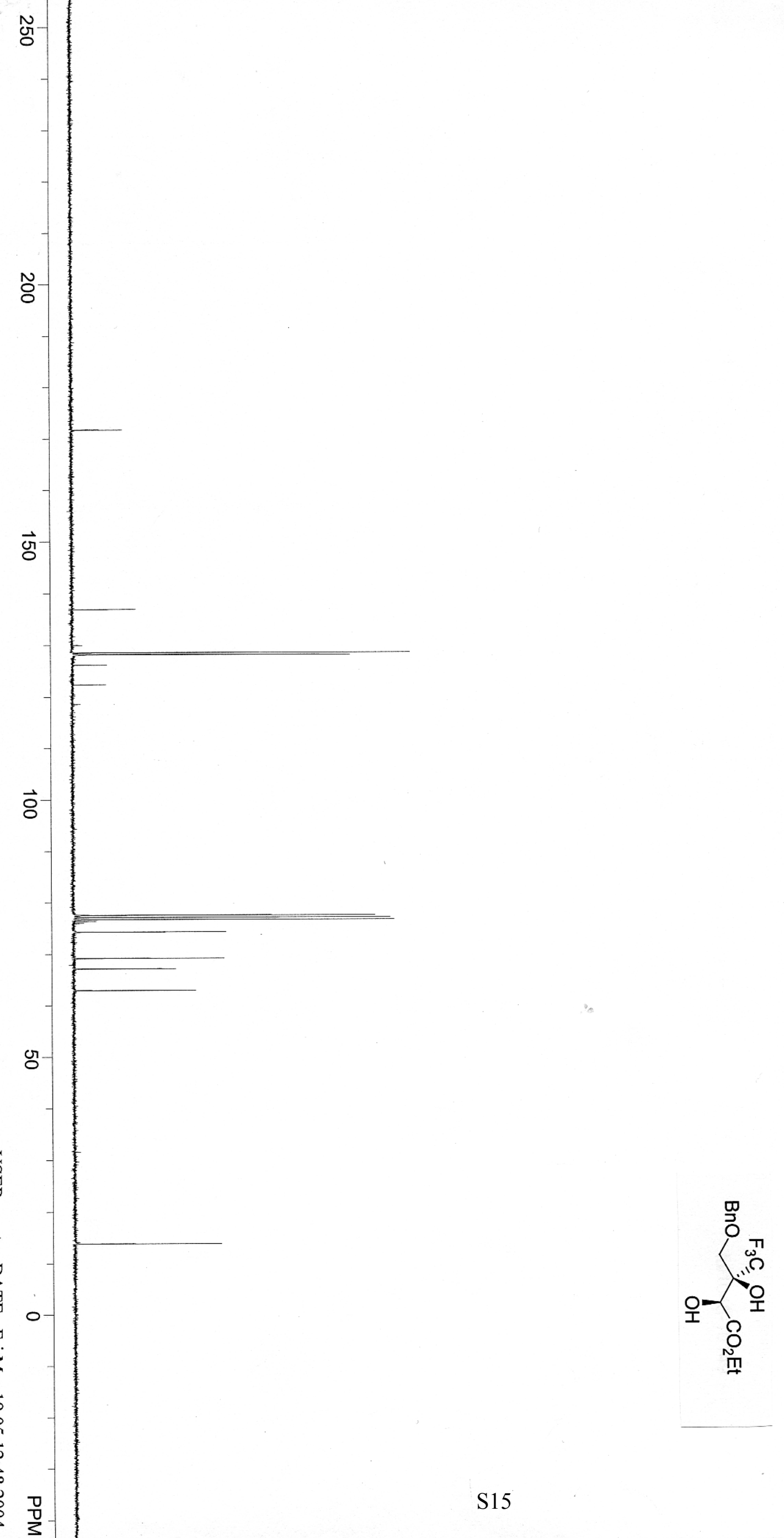

लू
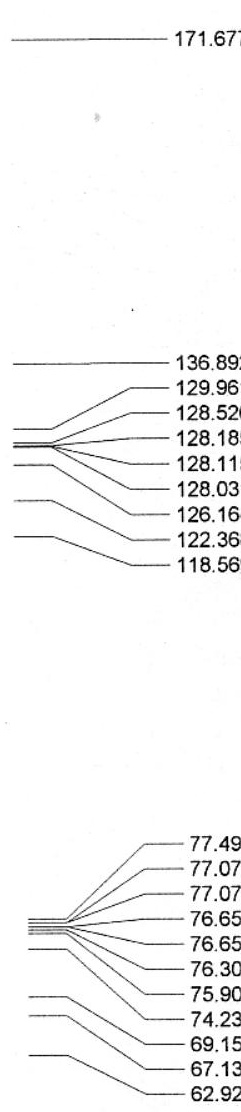

$\sum$ 

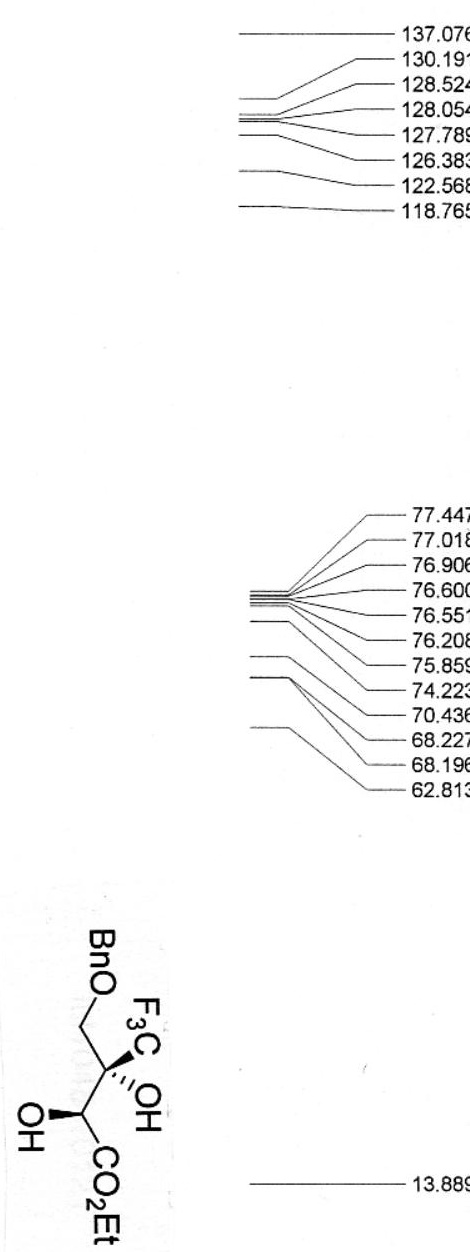

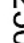
$-171.44$ 
光

等

$\vec{\circ}$

$\vec{b}$

$\overrightarrow{8}$

ह

\&

$\vec{N}-$

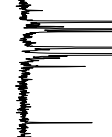

:<smiles>OCC(O)C1(O)CO[Se][Se]1</smiles>

\section{7

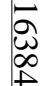

당

중

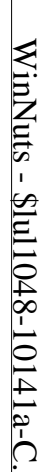

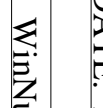

$-\frac{1}{3}$

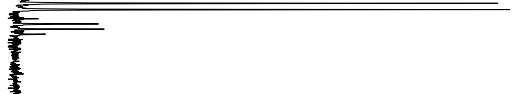

8

$-\frac{}{4}$

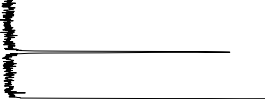




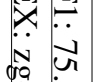

Oे

ำ<smiles>[O]CC(O)C(O)(O)CO</smiles>

$\overrightarrow{8}$

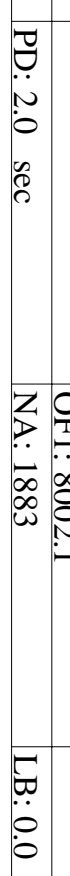

जु

in

엉-

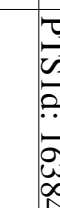

㩊 



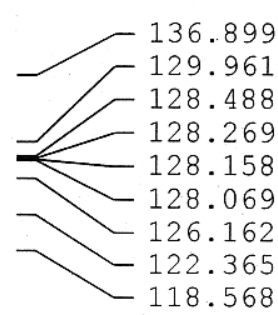

$\vec{\circ}$

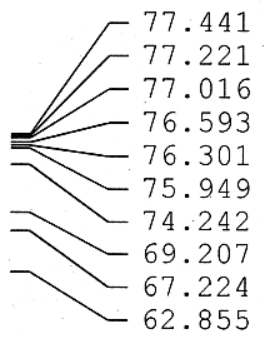

8
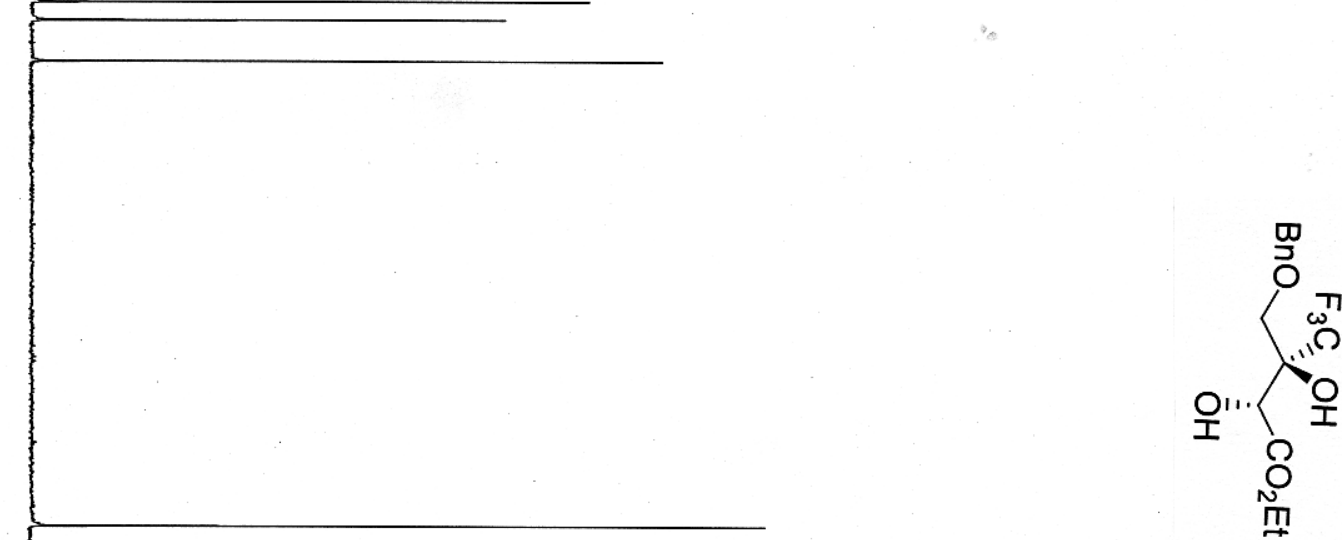


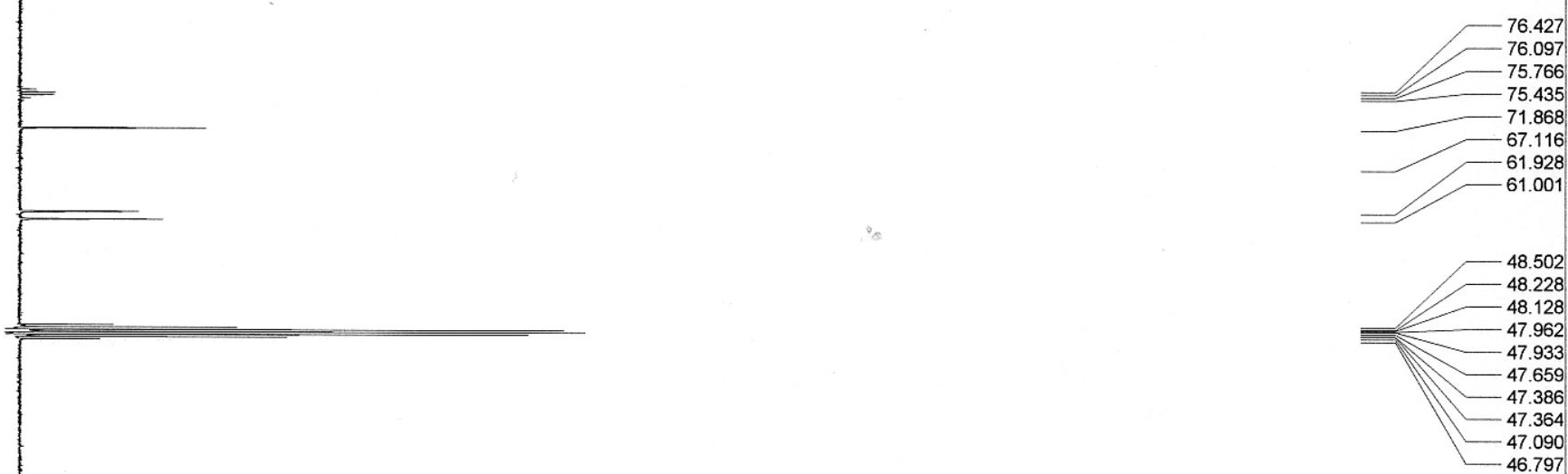


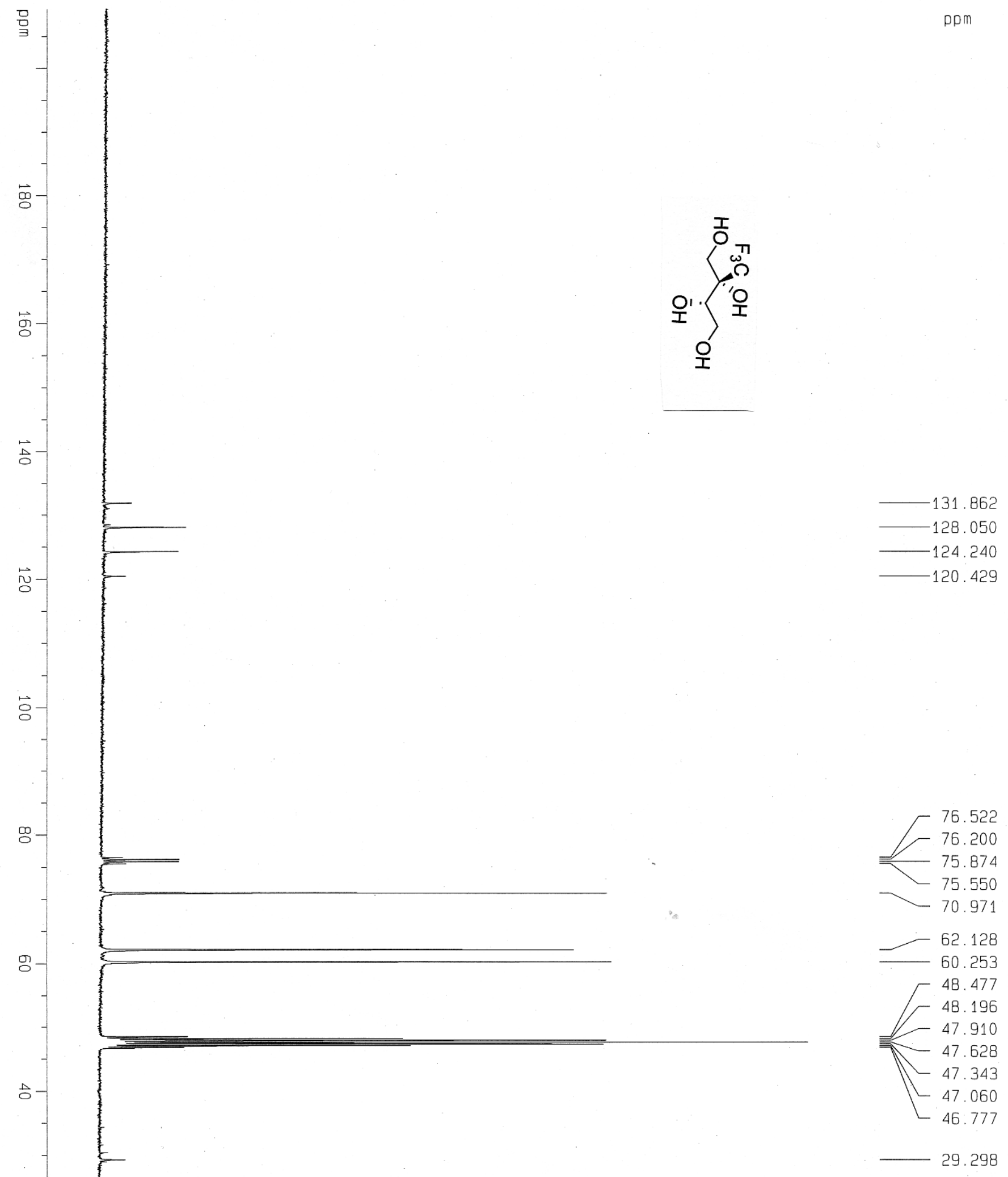




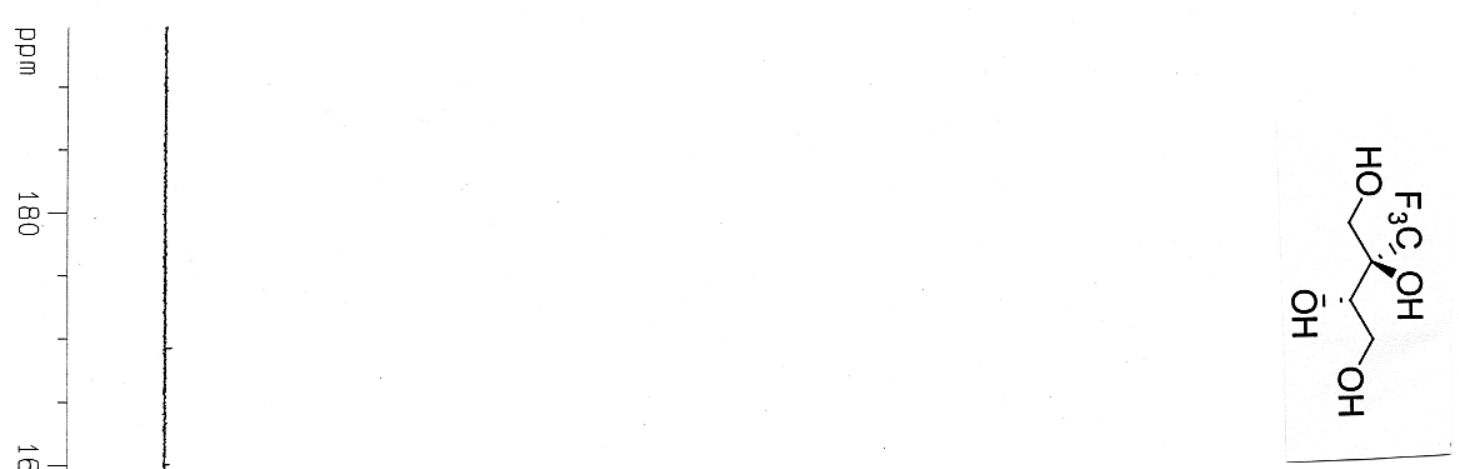




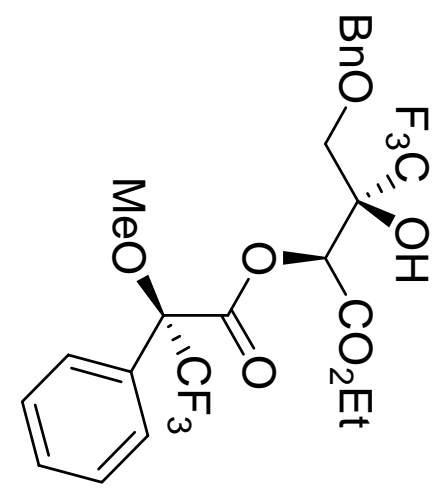




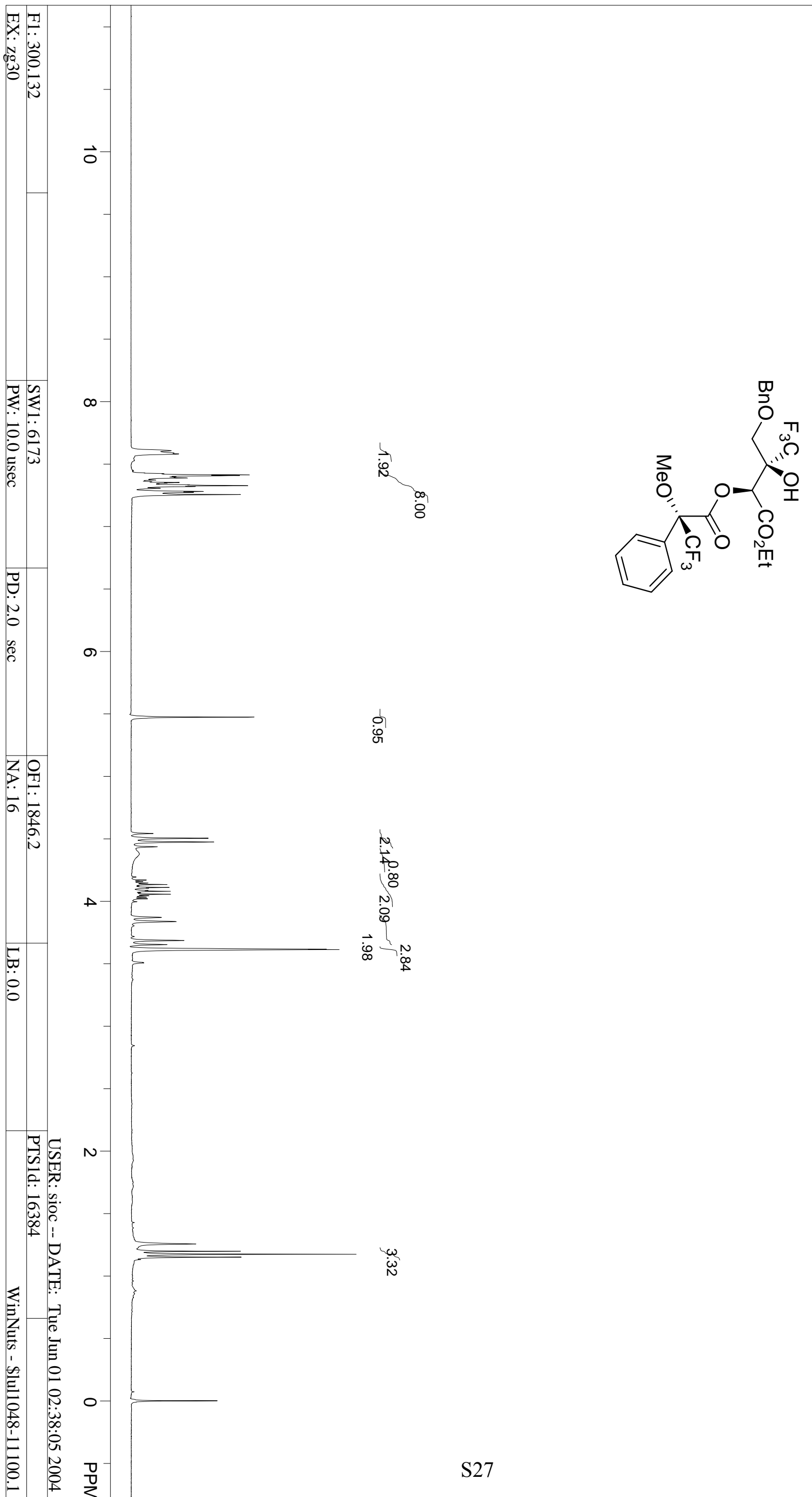




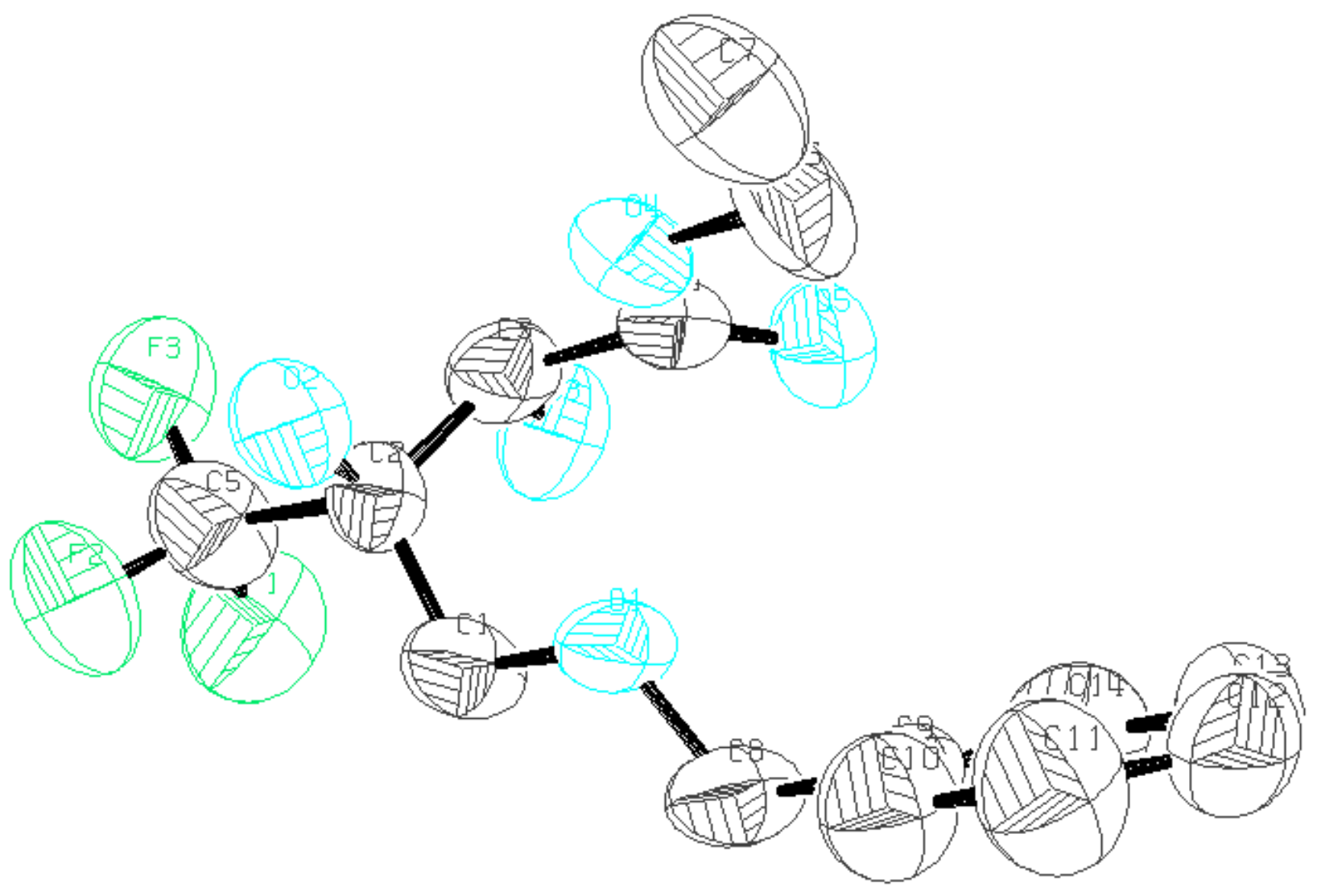

